\title{
GENERALIZED TRANSFORMS AND CONVOLUTIONS
}

\author{
TIMOTHY HUFFMAN \\ Department of Mathematics \\ Northwestern College \\ Orange City, IA 51041 \\ CHULL PARK \\ Department of Mathematics and Statistics \\ Miami University \\ Oxford, OH 45056 \\ DAVID SKOUG \\ Department of Mathematics and Statistics \\ University of Nebraska \\ Lincoln, NE 68588
}

(Received June 27, 1995 and in revised form August 8, 1995)

ABSTRACT. In this paper, using the concept of a generalized Feynman integral, we define a generalized Fourier-Feynman transform and a generalized convolution product. Then for two classes of functionals on Wiener space we obtain several results involving and relating these generalized transforms and convolutions. In particular we show that the generalized transform of the convolution product is a product of transforms. In addition we establish a Parseval's identity for functionals in each of these classes.

KEY WORDS AND PHRASES. Fourier-Feynman transform, convolution, Feynman integral.

1991 AMS SUBJECT CLASSIFICATION CODE. 28C20

\section{INTRODUCTION.}

The concept of an $\mathrm{L}_{1}$ analytic Fourier-Feynman transform (FFT) was introduced by Brue in [1]. In [2], Cameron and Storvick introduced an $\mathrm{L}_{2}$ analytic FFT. In [3], Johnson and Skoug developed an $\mathrm{L}_{\mathrm{p}}$ analytic FFT for $1 \leq \mathrm{p} \leq 2$ which extended the results in $[1,2]$ and gave various relationships between the $L_{1}$ and $L_{2}$ theories. In [4], Huffman, Park and Skoug defined a convolution product for functionals on Wiener space and in $[4,5]$ obtained various results involving the FFT and the convolution product. 
Both the FFT and the convolution product are defined in terms of a Feynman integral. In this paper we use the concept of the generalized Feynman integral, introduced by Chung, Park and Skoug in [6] and further developed in [7], to define a generalized FFT(GFFT) and a generalized convolution product (GCP).

In section 3 we establish several results involving and relating the GFFT and the GCP for functionals $F$ and $G$ in the Banach algebra $\mathscr{\varphi}$ introduced by Cameron and Storvick in [8]. In sections 4 and 5 we obtain similar results for a class $A_{n}^{(p)}$ of tame functionals. In particular we establish a Parseval's identity for functionals in each of these two classes.

In defining the FFT $[1,2,3]$ of $F$ and the convolution product [4] of $F$ and $G$, one starts with, for $\lambda>0$, the Wiener integrals

$$
\int_{C_{0}[0, T]} F\left(y+\lambda^{\left.-\frac{1}{2} x\right) m(d x)}\right.
$$

and

$$
\int_{0}[0, T] \quad F\left[\frac{y+\lambda^{-\frac{1}{2}} x}{\sqrt{2}}\right] G\left[\frac{y-\lambda^{-\frac{1}{2}} x}{\sqrt{2}}\right] m(d x)
$$

and then extends analytically in $\lambda$ to the right-half complex plane. In this paper, in defining the GFFT and the GCP we start with the Wiener integrals

$$
\begin{aligned}
& \int \mathrm{F}\left(\mathrm{y}+\lambda^{-\frac{1}{2}} \mathrm{Z}(\mathrm{x}, \cdot)\right) \mathrm{m}(\mathrm{dx}) \\
& \mathrm{C}_{0}[0, \mathrm{~T}]
\end{aligned}
$$

and

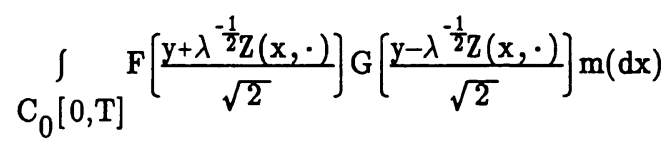

where $\mathrm{Z}$ is the Gaussian process

$$
Z(x, t)=\int_{0}^{t} h(s) d x(s)
$$

with $h$ in $L_{2}[0, T]$ and where $\int_{0}^{t} h(s) d x(s)$ denotes the Paley-Wiener-Zygmund stochastic integral.

\section{DEFINITIONS AND PRELIMINARIES.}

Let $\mathrm{C}_{0}[0, T]$ denote Wiener space; that is the space of all $\mathbb{R}$-valued continuous functions $\mathbf{x}(\mathrm{t})$ on $[0, \mathrm{~T}]$ with $\mathbf{x}(0)=0$. Let $\mathscr{N}$ denote the class of all Wiener measurable subsets of $\mathrm{C}_{0}[0, T]$ and let $m$ denote Wiener measure. $A$ subset $B$ of $C_{0}[0, T]$ is said to be scale-invariant measurable $[9,10]$ provided $\rho \mathrm{B} \in \mathcal{K}$ for all $\rho>0$, and a scale-invariant measurable set $\mathrm{N}$ is said to be scale-invariant null provided $\mathrm{m}(\rho \mathrm{N})=0$ for all $\rho>0$. A property that holds except on a scale-invariant null set is said to hold scale-invariant almost everywhere (s-a.e.). If two functionals $F$ and $G$ are equal s-a.e., we write $F \approx G$. Let $h$ be an element of $\mathrm{L}_{2}[0, T]$ with $\|\mathrm{h}\|>0$, let $\mathrm{Z}(\mathrm{x}, \mathrm{t})$ be given by (1.1), and let

$$
a(t)=\int_{0}^{t} h^{2}(u) d u .
$$


Then $\mathrm{Z}$ is a Gaussian process with mean zero and covariance function

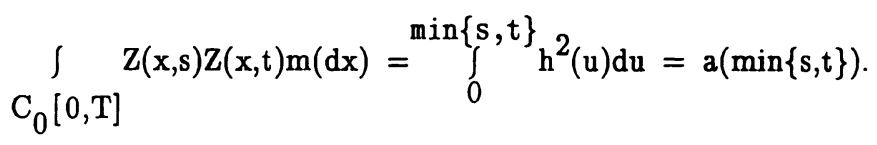

Next we state the definitions of the (generalized) analytic Feynman integral [6, p.388]. Let $\mathbb{C}_{+}=\{\lambda \in \mathbb{C}: \operatorname{Re} \lambda>0\}$ and let $\mathbb{C}_{+}^{-}=\{\lambda \in \mathbb{C}: \lambda \neq 0$ and $\operatorname{Re} \lambda \geq 0\}$. For each $\lambda>0$ assume that $\mathrm{F}\left(\lambda^{-\frac{1}{2}} \mathrm{Z}(\mathrm{x}, \cdot)\right)$ is Wiener integrable with respect to $\mathrm{x}$ on $\mathrm{C}_{0}[0, \mathrm{~T}]$, and let $\mathrm{J}(\lambda)=\int_{\mathrm{C}_{0}[0, T]} \mathrm{F}\left(\lambda^{\frac{-1}{2} \mathrm{Z}}(\mathrm{x}, \cdot)\right) \mathrm{m}(\mathrm{dx})$. If there exists a function $\mathrm{J}^{*}(\lambda)$ analytic on $\mathbb{C}_{+}$such that $\mathrm{J}^{*}(\lambda)=\mathrm{J}(\lambda)$ for $\lambda>0$, then we call $\mathrm{J}^{*}(\lambda)$ the (generalized) analytic Wiener integral of $F$ and for $\lambda \in \mathbb{C}_{+}$we write

$$
\left.\int_{\mathrm{C}_{0}[0, \mathrm{~T}]}^{\mathrm{anw}} \lambda \mathrm{Z}(\mathrm{x}, \cdot)\right) \mathrm{m}(\mathrm{dx})=\mathrm{J}^{*}(\lambda) .
$$

Let real $\mathrm{q} \neq 0$ be given. Then we define the (generalized) analytic Feynman integral of $F$ with parameter $q$ by $\left(\lambda \in \mathbf{C}_{+}\right)$

$$
\int_{\mathrm{C}_{0}[0, \mathrm{~T}]}^{\operatorname{anf}_{\mathrm{q}}} \mathrm{F}(\mathrm{Z}(\mathrm{x}, \cdot)) \mathrm{m}(\mathrm{dx})=\lim _{\lambda \rightarrow-\mathrm{iq}} \int_{\mathrm{C}_{0}[0, \mathrm{~T}]}^{\text {anw }_{\mathrm{N}}} \mathrm{F}(\mathrm{Z}(\mathrm{x}, \cdot)) \mathrm{m}(\mathrm{dx})
$$

if the limit exists.

We now proceed to define the GFFT and the GCP using the (generalized) analytic Wiener and Feynman integrals given by (2.2) and (2.3). For $\lambda \in \mathbb{C}_{+}$and y $\epsilon \mathrm{C}_{0}[0, \mathrm{~T}]$, let

$$
\left(\mathrm{T}_{\lambda}(\mathrm{F})\right)(\mathrm{y})=\int_{\mathrm{C}_{0}[0, \mathrm{~T}]}^{\mathrm{anw}} \lambda\left(\mathrm{y}+\lambda^{-\frac{1}{2}} \mathrm{Z}(\mathrm{x}, \cdot)\right) \mathrm{m}(\mathrm{dx}) .
$$

In the standard Fourier theory the integrals involved are often interpreted in the mean; a similar concept is useful in the FFT theory. Let $1<\mathrm{p} \leq 2$ be given and let $\mathrm{p}^{\prime}$ be determined by $\frac{1}{p}+\frac{1}{p^{\prime}}=1$. Let $\left\{H_{n}\right\}$ and $\mathrm{H}$ be scale-invariant measurable functionals such that for each $\rho>0$,

$$
\lim _{n \rightarrow \infty} \int_{C_{0}[0, T]}\left|H_{n}(\rho y)-H(\rho y)\right|^{p^{\prime}} m(d y)=0
$$

Then we write

$$
\text { 1.i.m. }\left(\mathrm{w}_{\mathrm{S}}^{\mathrm{p}^{\prime}}\right) \mathrm{H}_{\mathrm{n}} \approx \mathrm{H}
$$

and we call $H$ the scale invariant limit in the mean of order $\mathrm{p}^{\prime}$. A similar definition also applies for a continuous parameter $\lambda$.

DEFINITION. Let real $\mathrm{q} \neq 0$ be given. For $1<\mathrm{p} \leq 2$ we define the $\mathrm{L}_{\mathrm{p}}$ analytic GFFT, $\mathrm{T}_{\mathrm{q}}^{(\mathrm{p})}(\mathrm{F})$ of $\mathrm{F}$, by the formula $\left(\lambda \in \mathbb{C}_{+}\right)$

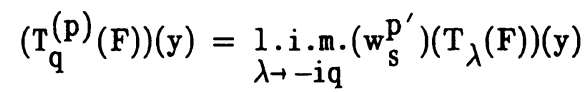

whenever this limit exists where $T_{\lambda}(F)$ is given by (2.4). We define the $L_{1}$ analytic GFFT, $\mathrm{T}_{\mathrm{q}}^{(1)}(\mathrm{F})$ of $\mathrm{F}$, by the formula $\left(\lambda \epsilon \mathbb{C}_{+}\right)$ 


$$
\left(\mathrm{T}_{\mathrm{q}}^{(1)}(\mathrm{F})\right)(\mathrm{y})=\lim _{\lambda \rightarrow-\mathrm{iq}}\left(\mathrm{T}_{\lambda}(\mathrm{F})\right)(\mathrm{y})
$$

for s-a.e. $y$. We note that for $1 \leq \mathrm{p} \leq 2, \mathrm{~T}_{\mathrm{q}}^{(\mathrm{p})}(\mathrm{F})$ is defined only s-a.e. We also note that if $\mathrm{T}_{\mathrm{q}}^{(\mathrm{p})}(\mathrm{F})$ exists and $\mathrm{F} \approx \mathrm{G}$, then $\mathrm{T}_{\mathrm{q}}^{(\mathrm{p})}(\mathrm{G})$ exists and $\mathrm{T}_{\mathrm{q}}^{(\mathrm{p})}(\mathrm{G}) \approx \mathrm{T}_{\mathrm{q}}^{(\mathrm{p})}(\mathrm{F})$.

DEFINITION. Let $F$ and $G$ be scale-invariant measurable functionals on $C_{0}[0, T]$. For $\lambda \in \mathbb{C}_{+}^{\sim}$ we define their GCP, $(F * G){ }_{\lambda}$ (if it exists) by

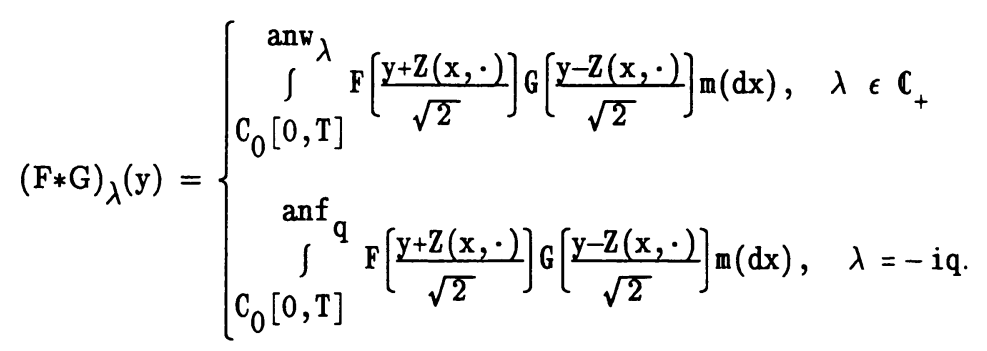

REMARKS. i) When $\lambda=-\mathrm{iq}$, we denote $(\mathrm{F} * \mathrm{G})_{\lambda}$ by $(\mathrm{F} * \mathrm{G})_{\mathrm{q}}$.

ii) When $h \equiv 1$ on $[0, T]$, then $Z(x, t)=x(t)$ and so the GFFT and the GCP reduce to the ordinary Fourier-Feynman transform and convolution product [4].

The following well-known Wiener integration formula

$$
\left.\int_{0}[0, T] \exp _{0} \int_{0}^{\mathrm{T}} \mathrm{g}(\mathrm{t}) \mathrm{dx}(\mathrm{t})\right\} \mathrm{m}(\mathrm{dx})=\exp \left\{-\frac{1}{2} \int_{0}^{\mathrm{T}} \mathrm{g}^{2}(\mathrm{t}) \mathrm{dt}\right\}, \mathrm{g} \in \mathrm{L}_{2}[0, \mathrm{~T}]
$$

is used several times in sections 3 and 5 .

We conclude this section with a theorem which play a key role in sections 3 and 5. THEOREM 2.1. If $\mathrm{T}_{\lambda}(\mathrm{F}), \mathrm{T}_{\lambda}(\mathrm{G})$ and $\mathrm{T}_{\lambda}(\mathrm{F} * \mathrm{G})_{\lambda}$ exist for $\lambda>0$, then

$$
\left(\mathrm{T}_{\lambda}(\mathrm{F} * \mathrm{G}){ }_{\lambda}\right)(\mathrm{y})=\left(\mathrm{T}_{\lambda}(\mathrm{F})\right)(\mathrm{y} / \sqrt{2})\left(\mathrm{T}_{\lambda}(\mathrm{G})\right)(\mathrm{y} / \sqrt{2})
$$

for all y $\in \mathrm{C}_{0}[0, \mathrm{~T}]$.

PROOF. For $\lambda>0$, using (2.4) and (2.7) we see that

$$
\begin{aligned}
& \left(\mathrm{T}_{\lambda}(\mathrm{F} * \mathrm{G})_{\lambda}\right)(\mathrm{y})=\int_{\mathrm{C}_{0}[0, \mathrm{~T}]}(\mathrm{F} * \mathrm{G})_{\lambda}\left(\mathrm{y}+\lambda^{-\frac{1}{2}} \mathrm{Z}\left(\mathrm{x}_{1}, \cdot\right)\right) \mathrm{m}\left(\mathrm{dx}_{1}\right) \\
& =\int_{\mathrm{C}_{0}^{2}[0, T]} \mathrm{F}\left[\frac{\mathrm{y}+\lambda^{-\frac{1}{2}}\left[\mathrm{Z}\left(\mathrm{x}_{1}, \cdot\right)+\mathrm{Z}\left(\mathrm{x}_{2}, \cdot\right)\right]}{\sqrt{2}}\right] \mathrm{G}\left[\frac{\mathrm{y}+\lambda^{-\frac{1}{2}}\left[\mathrm{Z}\left(\mathrm{x}_{1}, \cdot\right)-\mathrm{Z}\left(\mathrm{x}_{2}, \cdot\right)\right]}{\sqrt{2}}\right] \mathrm{m}\left(\mathrm{dx} \mathrm{x}_{1}\right) \mathrm{m}\left(\mathrm{dx_{2 }}\right) \\
& =\mathrm{C}_{0}^{2}[0, \mathrm{~T}] \\
& \mathrm{F}\left[\frac{\mathrm{y}}{\sqrt{2}}+\int_{0} \mathrm{~h}(\mathrm{t}) \mathrm{d}\left[\frac{\mathrm{x}_{1}(\mathrm{t})+\mathrm{x}_{2}(\mathrm{t})}{\sqrt{2 \lambda}}\right]\right] G\left[\frac{\mathrm{y}}{\sqrt{2}}+\int_{0} \mathrm{~h}(\mathrm{t}) \mathrm{d}\left[\frac{\mathrm{x}_{1}(\mathrm{t})-\mathrm{x}_{2}(\mathrm{t})}{\sqrt{2 \lambda}}\right]\right] \mathrm{m}\left(\mathrm{dx} \mathrm{x}_{1}\right) \mathrm{m}\left(\mathrm{dx} \mathrm{x}_{2}\right) .
\end{aligned}
$$

But $\mathrm{w}_{1}=\frac{\mathrm{x}_{1}+\mathrm{x}_{2}}{\sqrt{2}}$ and $\mathrm{w}_{2}=\frac{\mathrm{x}_{1}-\mathrm{x}_{2}}{\sqrt{2}}$ are independent standard Wiener processes and hence

$$
\begin{aligned}
\left(\mathrm{T}_{\lambda}(\mathrm{F} * \mathrm{G})_{\lambda}\right)(\mathrm{y}) & =\int_{\mathrm{C}_{0}[0, \mathrm{~T}]} \mathrm{F}\left(\frac{\mathrm{y}}{\sqrt{2}}+\lambda^{\frac{-1}{2}} \mathrm{Z}\left(\mathrm{w}_{1}, \cdot\right)\right) \mathrm{m}\left(\mathrm{dw}_{1}\right) \int_{\mathrm{C}_{0}[0, T]} \mathrm{G}\left(\frac{\mathrm{y}}{\sqrt{2}}+\lambda^{-\frac{\mathrm{i}}{2}} \mathrm{Z}\left(\mathrm{w}_{2}, \cdot\right)\right) \mathrm{m}\left(\mathrm{dw}_{2}\right) \\
& =\left(\mathrm{T}_{\lambda}(\mathrm{F})\right)(\mathrm{y} / \sqrt{2})\left(\mathrm{T}_{\lambda}(\mathrm{G})\right)(\mathrm{y} / \sqrt{2}) .
\end{aligned}
$$




\section{THE BANACH ALGEBRA $\mathscr{\varphi}$.}

The Banach algebra $\mathscr{f}$, see [8], consists of functionals expressible in the form

$$
F(x)=\int_{L_{2}[0, T]} \exp \left\{i \int_{0}^{T} v(t) d x(t)\right\} d f(v)
$$

for s-a.e. $x$ in $C_{0}[0, T]$ where $f$ is an element of $M\left(L_{2}[0, T]\right)$, the space of all $C$-valued countably additive finite Borel measures on $\mathrm{L}_{2}[0, \mathrm{~T}]$.

From [6, Lemma 1] we have that for each $v \in \mathrm{L}_{2}[0, T]$ and each $h \in \mathrm{L}_{\infty}[0, \mathrm{~T}]$,

$$
\int_{0}^{\mathrm{T}} \mathrm{v}(\mathrm{s}) \mathrm{dZ}(\mathrm{x}, \mathrm{s})=\int_{0}^{\mathrm{T}} \mathrm{v}(\mathrm{s}) \mathrm{d}\left[\int_{0}^{\mathrm{s}} \mathrm{h}(\mathrm{u}) \mathrm{dx}(\mathrm{u})\right]=\int_{0}^{\mathrm{T}} \mathrm{v}(\mathrm{s}) \mathrm{h}(\mathrm{s}) \mathrm{dx}(\mathrm{s})
$$

for s-a.e. $x$ in $C_{0}[0, T]$. Thus, throughout this section we require $h$ to be in $L_{\infty}[0, T]$, rather than simply in $\mathrm{L}_{2}[0, \mathrm{~T}]$.

THEOREM 3.1. Let $\mathrm{F} \in \mathscr{\mathscr { C }}$ be given by (3.1) and let $\mathrm{h} \in \mathrm{L}_{\infty}[0, \mathrm{~T}]$. Then for all $\mathrm{p} \in[1,2]$, the GFFT $T_{q}^{(p)}(F)$ exists for all real $q \neq 0$ and is given by

$$
\left(\mathrm{T}_{\mathrm{q}}^{(\mathrm{p})}(\mathrm{F})\right)(\mathrm{y})=\int_{\mathrm{L}_{2}[0, T]} \exp \left\{\mathrm{i} \int_{0}^{\mathrm{T}} \mathrm{v}(\mathrm{t}) \mathrm{dy}(\mathrm{t})-\frac{\mathrm{i}}{2 \mathrm{q}} \int_{0}^{\mathrm{T}} \mathrm{v}^{2}(\mathrm{t}) \mathrm{h}^{2}(\mathrm{t}) \mathrm{dt}\right\} \mathrm{df}(\mathrm{v}) .
$$

PROOF. First of all, using the Fubini theorem, (3.2) and (2.8), we see that for all $\lambda>0$ and s-a.e. $\mathrm{y}$ in $\mathrm{C}_{0}[0, \mathrm{~T}]$,

$$
\begin{aligned}
\left(\mathrm{T}_{\lambda}(\mathrm{F})\right)(\mathrm{y}) & =\int_{\mathrm{C}_{0}[0, \mathrm{~T}]} \mathrm{F}\left(\mathrm{y}+\lambda^{-\frac{1}{2}} \mathrm{Z}(\mathrm{x}, \cdot)\right) \mathrm{m}(\mathrm{dx}) \\
& =\int_{\mathrm{C}_{0}[0, \mathrm{~T}] \mathrm{L}_{2}[0, \mathrm{~T}]} \exp \left\{\mathrm{i} \int_{0}^{\mathrm{T}} \mathrm{v}(\mathrm{t}) \mathrm{d}\left[\mathrm{y}(\mathrm{t})+\lambda^{-\frac{1}{2}} \mathrm{Z}(\mathrm{x}, \mathrm{t})\right]\right\} \mathrm{df}(\mathrm{v}) \mathrm{m}(\mathrm{dx}) \\
& =\int_{\mathrm{L}_{2}[0, \mathrm{~T}] \mathrm{C}_{0}[0, \mathrm{~T}]} \exp \left\{\mathrm{i} \int_{0}^{\mathrm{T}} \mathrm{v}(\mathrm{t}) \mathrm{dy}(\mathrm{t})+\frac{\mathrm{i}}{\sqrt{\lambda}} \cdot \int_{0}^{\mathrm{T}} \mathrm{v}(\mathrm{t}) \mathrm{h}(\mathrm{t}) \mathrm{dx}(\mathrm{t})\right\} \mathrm{m}(\mathrm{dx}) \mathrm{df}(\mathrm{v}) \\
& =\int_{\mathrm{L}_{2}[0, \mathrm{~T}]} \exp \left\{\mathrm{i} \int_{0}^{\mathrm{T}} \mathrm{v}(\mathrm{t}) \mathrm{dy}(\mathrm{t})-\frac{1}{2 \lambda} \int_{0}^{\mathrm{T}} \mathrm{v}^{2}(\mathrm{t}) \mathrm{h}^{2}(\mathrm{t}) \mathrm{dt}\right\} \mathrm{df}(\mathrm{v})
\end{aligned}
$$

But the last expression above is an analytic function of $\lambda$ throughout $\mathbb{C}_{+}$and is a bounded continuous function of $\lambda$ on $\mathbf{C}_{+}^{\sim}$ since $f$ is a finite Borel measure. Hence $T_{q}^{(p)}(F)$ exists and is given by (3.3) for all $p \in[1,2]$ and all real $q \neq 0$.

THEOREM 3.2. Let $F$ and $G$ be elements of $\mathscr{f}$ with corresponding finite Borel measures $f$ and $g$ in $M\left(L_{2}[0, T]\right)$. Then their GCP $(F * G)_{q}$ exists for all real $q \neq 0$ and is given by the formula

$$
\begin{aligned}
(F * G)_{q}(y)= & \int_{L_{2}^{2}[0, T]} \exp \left\{\frac{i}{\sqrt{2}} \int_{0}^{T}[v(t)+w(t)] d y(t)\right\} \\
& \cdot \exp \left\{-\frac{i}{4 q} \int_{0}^{T} h^{2}(t)[v(t)-w(t)]^{2} d t\right\} d f(v) d g(w)
\end{aligned}
$$


PROOF. Proceeding as in the proof of Theorem 3.1 above, we obtain for all $\lambda>0$ and s-a.e. $y$ in $\mathrm{C}_{0}[0, \mathrm{~T}]$,

$$
\begin{aligned}
& (F * G){ }_{\lambda}(y)=\int_{C_{0}[0, T]} F\left[\frac{y+\lambda^{-\frac{1}{2}} Z(x, \cdot)}{\sqrt{2}}\right] G\left[\frac{y-\lambda^{-\frac{1}{2}} Z(x, \cdot)}{\sqrt{2}}\right] m(d x) \\
& =\int_{C_{0}[0, T] L_{2}[0, T]} \exp \left\{\frac{i}{\sqrt{2}} \int_{0}^{T} v(t) d y(t)+\frac{i}{\sqrt{2 \lambda}} \int_{0}^{T} v(t) h(t) d x(t)\right\} d f(v) \\
& \int_{\mathrm{L}_{2}[0, T]} \exp \left\{\frac{\mathrm{i}}{\sqrt{2}} \int_{0}^{\mathrm{T}} \mathrm{w}(\mathrm{t}) \mathrm{dy}(\mathrm{t})-\frac{\mathrm{i}}{\sqrt{2 \lambda}} \int_{0}^{\mathrm{T}} \mathrm{w}(\mathrm{t}) \mathrm{h}(\mathrm{t}) \mathrm{dx}(\mathrm{t})\right\} \mathrm{dg}(\mathrm{w}) \mathrm{m}(\mathrm{dx}) \\
& =\int_{\mathrm{L}_{2}[0, \mathrm{~T}]} \exp \left\{\frac{\mathrm{i}}{\sqrt{2}} \int_{0}^{\mathrm{T}}[\mathrm{v}(\mathrm{t})+\mathrm{w}(\mathrm{t})] \mathrm{dy}(\mathrm{t})\right\} \\
& \text { - } \exp \left\{-\frac{1}{4 \lambda} \int_{0}^{\mathrm{T}}[\mathrm{v}(\mathrm{t})-\mathrm{w}(\mathrm{t})]^{2} \mathrm{~h}^{2}(\mathrm{t}) \mathrm{dt}\right\} \mathrm{df}(\mathrm{v}) \mathrm{dg}(\mathrm{w}) .
\end{aligned}
$$

Again, (3.5) follows from (3.6) in the usual way by analytic continuation in $\lambda$.

Our next theorem shows that the GFFT of the GCP is a product of GFFT's.

THEOREM 3.3. Let $F, G, f$ and $g$ be as in Theorem 3.2. Then for all $p \in[1,2]$ and all real $q \neq 0$,

$$
\left(\mathrm{T}_{\mathrm{q}}^{(\mathrm{p})}(\mathrm{F} * \mathrm{G})_{\mathrm{q}}\right)(\mathrm{y})=\left(\mathrm{T}_{\mathrm{q}}^{(\mathrm{p})}(\mathrm{F})\right)(\mathrm{y} / \sqrt{2})\left(\mathrm{T}_{\mathrm{q}}^{(\mathrm{p})}(\mathrm{G})\right)(\mathrm{y} / \sqrt{2}) .
$$

PROOF. By Theorem 2.1 we see that

$$
\left(\mathrm{T}_{\lambda}(\mathrm{F} * \mathrm{G})_{\lambda}\right)(\mathrm{y})=\left(\mathrm{T}_{\lambda}(\mathrm{F})\right)(\mathrm{y} / \sqrt{2})\left(\mathrm{T}_{\lambda}(\mathrm{G})\right)(\mathrm{y} / \sqrt{2})
$$

holds for all $\lambda>0$. But both of the expressions on the right-hand side of equation (3.8) are analytic functions of $\lambda$ throughout $\mathbf{C}_{+}$, and are bounded continuous functions of $\lambda$ on $\mathbb{C}_{+}^{\sim}$ for all $y \in C_{0}[0, T]$. Hence $T_{q}^{(p)}(F * G)_{q}$ exists and is given by (3.7) for all desired values of $p$ and $q$.

In our next theorem we establish a Parseval's identity for functionals in the Banach algebra $\mathscr{f}$.

THEOREM 3.4. Let $\mathrm{F}$ and $\mathrm{G}$ be as in Theorem 3.2. Then the Parseval's identity

$$
\begin{gathered}
\int_{0}^{\operatorname{anf}}\left(\mathrm{T}_{\mathrm{q}}^{(\mathrm{p})}(\mathrm{F})\right)\left[\frac{\mathrm{Z}(\mathrm{x}, \cdot)}{\sqrt{2}}\right]\left(\mathrm{T}_{\mathrm{q}}^{(\mathrm{p})}(\mathrm{G})\right)\left[\frac{\mathrm{Z}(\mathrm{x}, \cdot)}{\sqrt{2}}\right] \mathrm{m}(\mathrm{dx}) \\
\quad=\int_{\mathrm{C}_{0}[0, \mathrm{~T}]}^{\mathrm{anf}} \mathrm{F}\left[\frac{\mathrm{Z}(\mathrm{x}, \cdot)}{\sqrt{2}}\right] \mathrm{G}\left[-\frac{\mathrm{Z}(\mathrm{x}, \cdot)}{\sqrt{2}}\right] \mathrm{m}(\mathrm{dx})
\end{gathered}
$$

holds for all real $\mathrm{q} \neq 0$ and all $\mathrm{p} \in[1,2]$.

PROOF. First of all for $\lambda>0$, we see that 


$$
\begin{aligned}
& \int_{\mathrm{C}_{0}[0, \mathrm{~T}]}\left(\mathrm{T}_{\mathrm{q}}^{(\mathrm{p})}(\mathrm{F})\right)\left[\frac{\mathrm{Z}(\mathrm{x}, \cdot)}{\sqrt{2 \lambda}}\right]\left(\mathrm{T}_{\mathrm{q}}^{(\mathrm{p})}(\mathrm{G})\right)\left[\frac{\mathrm{Z}(\mathrm{x}, \cdot)}{\sqrt{2 \lambda}}\right] \mathrm{m}(\mathrm{dx}) \\
& =\int_{\mathrm{C}_{0}[0, \mathrm{~T}] \mathrm{L}_{2}[0, \mathrm{~T}]} \exp \left\{-\frac{\mathrm{i}}{2 \mathrm{q}} \int_{0}^{\mathrm{T}} \mathrm{v}^{2}(\mathrm{t}) \mathrm{h}^{2}(\mathrm{t}) \mathrm{dt}+\frac{\mathrm{i}}{\sqrt{2 \lambda}} \int_{0}^{\mathrm{T}} \mathrm{v}(\mathrm{t}) \mathrm{h}(\mathrm{t}) \mathrm{dx}(\mathrm{t})\right\} \mathrm{df}(\mathrm{v}) \\
& \text {. } \int_{\mathrm{L}_{2}[0, T]} \exp \left\{-\frac{\mathrm{i}}{2 \mathrm{q}} \int_{0}^{\mathrm{T}} \mathrm{w}^{2}(\mathrm{t}) \mathrm{h}^{2}(\mathrm{t}) \mathrm{dt}+\frac{\mathrm{i}}{\sqrt{2 \lambda}} \int_{0}^{\mathrm{T}} \mathrm{w}(\mathrm{t}) \mathrm{h}(\mathrm{t}) \mathrm{dx}(\mathrm{t})\right\} \mathrm{dg}(\mathrm{w}) \mathrm{m}(\mathrm{dx}) \\
& =\int_{\mathrm{L}_{2}^{2}[0, \mathrm{~T}]} \exp \left\{-\frac{\mathrm{i}}{2 \mathrm{q}} \int_{0}^{\mathrm{T}} \mathrm{h}^{2}(\mathrm{t})\left[\mathrm{v}^{2}(\mathrm{t})+\mathrm{w}^{2}(\mathrm{t})\right] \mathrm{dt}\right\} \\
& \text { - } \exp \left\{-\frac{1}{4 \lambda} \int_{0}^{\mathrm{T}} \mathrm{h}^{2}(\mathrm{t})[\mathrm{v}(\mathrm{t})+\mathrm{w}(\mathrm{t})]^{2} \mathrm{dt}\right\} \mathrm{df}(\mathrm{v}) \mathrm{dg}(\mathrm{w}) .
\end{aligned}
$$

But the last expression above is a continuous function of $\lambda$ on $\boldsymbol{C}_{+}^{\sim}$ and so setting $\lambda=-(-\mathrm{iq})=\mathrm{iq}$ we obtain that

$$
\begin{aligned}
& \int_{C_{0}[0, T]}^{\operatorname{anf}}\left(T_{q}^{(p)}(F)\right)\left[\frac{Z(x, \cdot)}{\sqrt{2}}\right]\left(T_{q}^{(p)}(G)\right)\left[\frac{Z(x, \cdot)}{\sqrt{2}}\right] m(d x) \\
& \quad=\int_{L_{2}^{2}[0, T]} \exp \left\{-\frac{i}{4 q} \int_{0}^{T} h^{2}(t)[v(t)-w(t)]^{2} d t\right\} d f(v) d g(w) .
\end{aligned}
$$

Next for $\lambda>0$, we see that

$$
\begin{aligned}
& \int_{\mathrm{C}_{0}[0, T]} F\left[\frac{\mathrm{Z}(\mathrm{x}, \cdot)}{\sqrt{2 \lambda}}\right] \mathrm{G}\left[-\frac{\mathrm{Z}(\mathrm{x}, \cdot)}{\sqrt{2 \lambda}}\right] \mathrm{m}(\mathrm{dx}) \\
& =\int_{\mathrm{C}_{0}[0, \mathrm{~T}] \mathrm{L}_{2}^{2}[0, \mathrm{~T}]} \exp \left\{\frac{\mathrm{i}}{\sqrt{2 \lambda}} \int_{0}^{\mathrm{T}} \mathrm{h}(\mathrm{t})[\mathrm{v}(\mathrm{t})-\mathrm{w}(\mathrm{t})] \mathrm{dx}(\mathrm{t})\right\} \mathrm{df}(\mathrm{v}) \mathrm{dg}(\mathrm{w}) \mathrm{m}(\mathrm{dx}) \\
& =\int_{\mathrm{L}_{2}^{2}[0, T]}^{\int} \exp \left\{-\frac{1}{4 \lambda} \int_{0}^{\mathrm{T}} \mathrm{h}^{2}(\mathrm{t})[\mathrm{v}(\mathrm{t})-\mathrm{w}(\mathrm{t})]^{2} \mathrm{dt}\right\} \mathrm{df}(\mathrm{v}) \mathrm{dg}(\mathrm{w}) .
\end{aligned}
$$

But the last expression above is a continuous function of $\lambda$ on $\mathbf{C}_{+}^{\sim}$ and so setting $\lambda=-i q$ we obtain that

$$
\begin{aligned}
& \quad \int_{\mathrm{C}_{0}[0, \mathrm{~T}]}^{\mathrm{anf}} \mathrm{F}\left[\frac{\mathrm{Z}(\mathrm{x}, \cdot)}{\sqrt{2}}\right] \mathrm{G}\left[-\frac{\mathrm{Z}(\mathrm{x}, \cdot)}{\sqrt{2}}\right] \mathrm{m}(\mathrm{dx}) \\
& \quad=\int_{\mathrm{L}_{2}^{2}[0, \mathrm{~T}]} \exp \left\{-\frac{\mathrm{i}}{4 \mathrm{q}} \int_{0}^{\mathrm{T}} \mathrm{h}^{2}(\mathrm{t})[\mathrm{v}(\mathrm{t})-\mathrm{w}(\mathrm{t})]^{2} \mathrm{dt}\right\} \mathrm{df}(\mathrm{v}) \mathrm{dg}(\mathrm{w}) .
\end{aligned}
$$

Now (3.10) and (3.11) together yield (3.9).

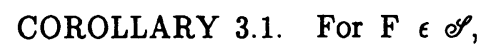

$$
\int_{\mathrm{C}_{0}[0, \mathrm{~T}]}^{\text {anf }^{-\mathrm{q}}}\left[\left(\mathrm{T}_{\mathrm{q}}^{(\mathrm{p})}(\mathrm{F})\right)\left[\frac{\mathrm{Z}(\mathrm{x}, \cdot)}{\sqrt{2}}\right)\right]^{2} \mathrm{~m}(\mathrm{dx})=\int_{\mathrm{C}_{0}[0, \mathrm{~T}]}^{\operatorname{anf}_{\mathrm{q}}} \mathrm{F}\left[\frac{\mathrm{Z}(\mathrm{x}, \cdot)}{\sqrt{2}}\right] \mathrm{F}\left[-\frac{\mathrm{Z}(\mathrm{x}, \cdot)}{\sqrt{2}}\right] \mathrm{m}(\mathrm{dx}) \text {, }
$$


(ii)

$$
\int_{\mathrm{C}_{0}[0, \mathrm{~T}]}^{\text {anf }}\left(\mathrm{T}_{\mathrm{q}}^{(\mathrm{p})}(\mathrm{F})\right)\left[\frac{\mathrm{Z}(\mathrm{x}, \cdot)}{\sqrt{2}}\right] \mathrm{m}(\mathrm{dx})=\int_{\mathrm{C}_{0}[0, \mathrm{~T}]}^{\operatorname{anf}_{\mathrm{q}}} \mathrm{F}\left[\frac{\mathrm{Z}(\mathrm{x}, \cdot)}{\sqrt{2}}\right] \mathrm{m}(\mathrm{dx}) \text {. }
$$

\section{GENERALIZED TRANSFORMS OF TAME FUNCTIONALS.}

Let $\mathrm{n}$ be a positive integer and let $0=\mathrm{t}_{0}<\mathrm{t}_{1}<\cdots<\mathrm{t}_{\mathrm{n}} \leq \mathrm{T}$. For $1 \leq \mathrm{p}<+\infty$ let $A_{n}^{(p)}$ be the space of all functionals $F$ on $C_{0}[0, T]$ of the form

$$
F(x)=f\left(x\left(t_{1}\right), \cdots, x\left(t_{n}\right)\right)
$$

s-a.e. where $f: \mathbb{R}^{n} \rightarrow \mathbb{C}$ is in $L_{p}\left(\mathbb{R}^{n}\right)$. Let $A_{n}^{(\infty)}$ be the space of all functionals of the form (4.1) with $f$ in $C_{0}\left(\mathbb{R}^{n}\right)$, the space of bounded continuous functions on $\mathbb{R}^{\mathrm{n}}$ that vanish at infinity.

In this section we don't need the added condition that $\mathrm{h} \in \mathrm{L}_{\mathrm{\omega}}[0, \mathrm{~T}]$; we only need require that $h \in \mathrm{L}_{2}[0, \mathrm{~T}]$. We will however, for convenience, assume that $h$ is such that

$$
0=a\left(t_{0}\right)<a\left(t_{1}\right)<\cdots<a\left(t_{n}\right) \leq a(T) .
$$

For if $a\left(t_{j-1}\right)=a\left(t_{j}\right)$ for some $j$, then in equation (4.4) below we would simply carry out the integration with respect to $\mathbf{v}_{\mathrm{j}}$ before making the substitution (4.5).

For notational purposes let $\Delta_{j} a \equiv a\left(t_{j}\right)-a\left(t_{j-1}\right)$ for $j=1,2, \cdots, n$ and let

$$
\gamma \equiv \prod_{j=1}^{n}\left[\frac{1}{2 \pi\left[a\left(t_{j}\right)-a\left(t_{j-1}\right)\right]}\right]^{\frac{1}{2}} .
$$

LEMMA 4.1. Let $1 \leq \mathrm{p} \leq+\infty$ and $\mathrm{F} \in \mathrm{A}_{\mathrm{n}}^{(\mathrm{p})}$ be given by (4.1). Then for all $\lambda \in \mathbf{C}_{+}$,

$$
\left(\mathrm{T}_{\lambda}(\mathrm{F})\right)(\mathrm{y})=\mathrm{K}\left(\lambda ; \mathrm{y}\left(\mathrm{t}_{1}\right), \cdots, \mathrm{y}\left(\mathrm{t}_{\mathbf{n}}\right)\right)
$$

where

$$
\begin{aligned}
& \mathrm{K}\left(\lambda ; \mathbf{w}_{1}, \cdots, \mathbf{w}_{\mathbf{n}}\right) \equiv \mathbf{K}(\lambda ; \overrightarrow{\mathbf{w}}) \\
& \equiv \lambda^{\frac{n}{2}} \gamma \int f(\vec{w}+\vec{u}) \exp \left\{-\frac{\lambda}{2} \sum_{j=1}^{n} \frac{\left(u_{j}-u_{j-1}\right)^{2}}{\Delta_{j}^{a}}\right\} d \vec{u} \\
& =\lambda^{\frac{n}{2}} \gamma \int \underset{\mathbb{R}^{n}}{f}(\vec{u}) \exp \left\{-\frac{\lambda}{2} \sum_{j=1}^{n} \frac{\left[\left(u_{j}-u_{j-1}\right)-\left(w_{j}-w_{j-1}\right)\right]^{2}}{\Delta_{j}^{a}}\right\} d \vec{u},
\end{aligned}
$$

and where $\overrightarrow{\mathrm{u}}=\left(\mathrm{u}_{1}, \cdots, \mathrm{u}_{\mathrm{n}}\right), \overrightarrow{\mathrm{w}}=\left(\mathrm{w}_{1}, \cdots, \mathrm{w}_{\mathrm{n}}\right)$ and $\mathrm{u}_{0}=0=\mathrm{w}_{0}$.

PROOF. For $\lambda>0$, we note that

$$
\begin{aligned}
& \left(T_{\lambda}(F)\right)(y)=\int_{C_{0}[0, T]} F\left(y+\lambda^{-\frac{1}{2}} Z(x, \cdot)\right) m(d x) \\
& =\int_{C_{0}[0, T]} f\left(y\left(t_{1}\right)+\lambda^{-\frac{1}{2}} \int_{0}^{t} h d x, \cdots, y\left(t_{n}\right)+\lambda^{-\frac{1}{2}} \int_{0}^{t} h d x\right) m(d x)
\end{aligned}
$$




$$
\begin{gathered}
=\int_{C_{0}[0, T]} f\left(y\left(t_{1}\right)+\lambda^{-\frac{1}{2}} \sum_{j=1}^{1} \int_{t_{j-1}}^{t_{j}} h d x, \cdots, y\left(t_{n}\right)+\lambda^{-\frac{1}{2}} \sum_{j=1}^{n} \int_{t_{j-1}}^{t_{j}} h d x\right) m(d x) \\
=\left[\frac{1}{2 \pi}\right]_{\mathbb{R}^{n}}^{\frac{n}{2}} f\left(y\left(t_{1}\right)+\lambda^{-\frac{1}{2}} \sum_{j=1}^{1}\left(\Delta_{j} a\right)^{\frac{1}{2}} v_{j}, \cdots, y\left(t_{n}\right)+\lambda^{-\frac{1}{2}} \sum_{j=1}^{n}\left(\Delta_{j} a\right)^{\frac{1}{2}} v_{j}\right) \exp \left\{-\frac{1}{2} \sum_{j=1}^{n} v_{j}^{2}\right\} d \vec{v} .
\end{gathered}
$$

Next for $\mathrm{j}=1,2, \cdots, \mathrm{n}$ let

$$
u_{j}=y\left(t_{j}\right)+\lambda^{-\frac{1}{2}} \sum_{k=1}^{j}\left(\Delta_{k} a\right)^{\frac{1}{2}} v_{k}
$$

Then for $\mathrm{j}=1,2, \cdots, \mathrm{n}, \mathrm{v}_{\mathrm{j}}=\lambda^{\frac{1}{2}}\left(\Delta_{\mathrm{j}} \mathrm{a}\right)^{\frac{-1}{2}}\left[\left(\mathrm{u}_{\mathrm{j}}-\mathrm{u}_{\mathrm{j}-1}\right)-\left(\mathrm{y}\left(\mathrm{t}_{\mathrm{j}}\right)-\mathrm{y}\left(\mathrm{t}_{\mathrm{j}-1}\right)\right]\right.$, and substituting into (4.4) yields

$$
\begin{aligned}
\left(T_{\lambda}(F)\right)(y) & =\lambda^{\frac{n}{2}} \gamma \int_{\mathbb{R}^{n}} f(\vec{u}) \exp \left\{-\frac{\lambda}{2} \sum_{j=1}^{n} \frac{\left[\left(u_{j}-u_{j-1}\right)-\left(y\left(t_{j}\right)-y\left(t_{j-1}\right)\right)\right]^{2}}{\Delta_{j}{ }^{2}}\right\} d \vec{u} \\
& =K\left(\lambda ; y\left(t_{1}\right), \cdots, y\left(t_{n}\right)\right) .
\end{aligned}
$$

Since $f \in L_{p}\left(\mathbb{R}^{n}\right)$ and $\operatorname{Re} \lambda>0$ for $\lambda \in \mathbb{C}_{+}$, the above expression is an analytic function of $\lambda$ throughout $\mathbb{C}_{+}$.

REMARK. In [3, pp.106-112], Johnson and Skoug established various results about the FFT for $F \in A_{n}^{(p)}$; ie, for the GFFT in the case $h \equiv 1$ on $[0, T]$. But their proofs can be adapted to work here, sometimes by just replacing $t_{j}-t_{j-1}$ with $a\left(t_{j}\right)-a\left(t_{j-1}\right)$. Thus we will simply state the generalized results below.

LEMMA 4.2. Let $1 \leq p \leq 2$, let $p^{\prime}$ be determined by $\frac{1}{p}+\frac{1}{p^{\prime}}=1$, and let $f \in A_{n}^{(p)}$. Then for all $\lambda \in \mathbb{C}_{+}^{\sim}, K(\lambda ; \vec{w})$ given by (4.3) is an element of $L_{p^{\prime}}\left(\mathbb{R}^{n}\right)$. Furthermore, when $\mathrm{p}=1, \mathrm{~K}(\lambda ; \overrightarrow{\mathrm{w}})$ is in $\mathrm{C}_{0}\left(\mathbb{R}^{\mathrm{n}}\right)$.

REMARK. When $1<\mathrm{p} \leq 2$ and $\operatorname{Re} \lambda=0$, the integral in (4.3) should be interpreted in the mean just as in the theory of the $\mathrm{L}_{p}$ Fourier transform.

THEOREM 4.1. Let $1 \leq \mathrm{p} \leq 2$ and let $F \in \mathrm{A}_{\mathrm{n}}^{(\mathrm{p})}$ be given by (4.1). Then the GFFT of $F, T_{q}^{(p)}(F)$ exists, is in $A_{n}^{\left(p^{\prime}\right)}$, and is given by

$$
\left(\mathrm{T}_{\mathrm{q}}^{(\mathrm{p})}(\mathrm{F})\right)(\mathrm{y})=\mathrm{K}\left(-\mathrm{iq} ; \mathrm{y}\left(\mathrm{t}_{1}\right), \cdots, \mathrm{y}\left(\mathrm{t}_{\mathrm{n}}\right)\right)
$$

for all real $q \neq 0$.

We also have the following transform theorems.

THEOREM 4.2. Let $1 \leq \mathrm{p} \leq 2$, real $\mathrm{q} \neq 0$, and $\mathrm{F} \in \mathrm{A}_{\mathrm{n}}^{(\mathrm{p})}$ be given. Then for each $\rho>0\left(\lambda \in \mathbb{C}_{+}\right)$,

$$
\lim _{\lambda \rightarrow-\mathrm{iq}} \int_{\mathrm{C}_{0}[0, \mathrm{~T}]}\left|\mathrm{T}_{\lambda}\left(\mathrm{T}_{\lambda}(\mathrm{F})\right)(\rho \mathrm{Z}(\mathrm{y}, \cdot))-\mathrm{F}(\rho \mathrm{Z}(\mathrm{y}, \cdot))\right|^{\mathrm{P}_{\mathrm{m}}(\mathrm{dy})=0 .}
$$

Furthermore,

$$
\mathrm{T}_{\lambda}\left(\mathrm{T}_{\lambda}(\mathrm{F})\right) \rightarrow \mathrm{F}
$$

s-a.e. as $\lambda \rightarrow-\mathrm{iq}$ through values in $\mathbf{C}_{+}$. 
THEOREM 4.3. Let $F \in A_{n}^{(2)}$. Then $T_{-q}\left(T_{q}(F)\right) \approx F$ for all real $q \neq 0$.

\section{GENERALIZED CONVOLUTION THEOREMS.}

Our first lemma gives an expression for the GCP $(F * G)_{\lambda}$ for $\lambda \in \mathbb{C}_{+}$where $F \in A_{n}^{\left(p_{1}\right)}$ is given by (4.1) and $G \in A_{n}^{\left(p_{2}\right)}$ is given by

$$
\mathrm{G}(\mathrm{x})=\mathrm{g}\left(\mathrm{x}\left(\mathrm{t}_{1}\right), \cdots, \mathrm{x}\left(\mathrm{t}_{\mathrm{n}}\right)\right)
$$

s-a.e. where $\mathrm{g}: \mathbb{R}^{\mathrm{n}} \rightarrow \mathbb{C}$ is in $\mathrm{L}_{\mathrm{p}_{2}}\left(\mathbb{R}^{\mathrm{n}}\right)$ and $0=\mathrm{t}_{0}<\mathrm{t}_{1}<\cdots<\mathrm{t}_{\mathrm{n}} \leq \mathrm{T}$.

LEMMA 5.1. Let $F \in A_{n}^{\left(p_{1}\right)}$ and $G \in A_{n}^{\left(p_{2}\right)}$ with $1 \leq p_{1} \leq+\infty$ and $1 \leq p_{2} \leq+\infty$. Then for all $\lambda \in \mathbb{C}_{+}$,

$$
(F * G)_{\lambda}(y)=H\left(\lambda ; y\left(t_{1}\right), \cdots, y\left(t_{n}\right)\right)
$$

where

$$
\begin{aligned}
& \mathrm{H}\left(\lambda ; \mathbf{w}_{1}, \cdots, \mathbf{w}_{\mathbf{n}}\right) \equiv \mathrm{H}(\lambda ; \overrightarrow{\mathbf{w}}) \\
& =\lambda^{\frac{n}{2}} \gamma \int_{\mathbb{R}^{n}} f\left[\frac{\overrightarrow{\mathrm{w}}+\overrightarrow{\mathrm{u}}}{\sqrt{2}}\right] \mathrm{g}\left[\frac{\overrightarrow{\mathrm{w}}-\overrightarrow{\mathrm{u}}}{\sqrt{2}}\right] \exp \left\{-\frac{\lambda}{2} \sum_{\mathrm{j}=1}^{\mathrm{n}} \frac{\left(\mathrm{u}_{\mathrm{j}}-\mathbf{u} j-1\right)^{2}}{\Delta_{j}^{\mathrm{a}}}\right\} \mathrm{d} \overrightarrow{\mathrm{u}} \text {. }
\end{aligned}
$$

PROOF. We first note that for $\lambda>0$,

$$
\begin{aligned}
& (F * G)_{\lambda}(y)=\int_{C_{0}[0, T]} F\left(\frac{y+\lambda^{-\frac{1}{2}} Z(x, \cdot)}{\sqrt{2}}\right) G\left(\frac{y-\lambda^{-\frac{1}{2}} Z(x, \cdot)}{\sqrt{2}}\right) m(d x) \\
& =\int_{C_{0}[0, T]} f\left(2^{-\frac{1}{2}} y\left(t_{1}\right)+(2 \lambda)^{-\frac{1}{2}} \int_{0}^{t_{1}} h d x, \cdots, 2^{-\frac{1}{2}} y\left(t_{n}\right)+(2 \lambda)^{-\frac{1}{2}} \sum_{j=1}^{n} \int_{t_{j-1}}^{t_{j}} h(s) d x(s)\right) \\
& \text { - } g\left(2^{-\frac{1}{2}} y\left(t_{1}\right)-(2 \lambda)^{-\frac{1}{2}} \int_{0}^{t_{1}} h d x, \cdots, 2^{-\frac{1}{2}} y\left(t_{n}\right)-(2 \lambda)^{-\frac{1}{2}} \sum_{j=1}^{n} \int_{t_{j-1}}^{t_{j}} h(s) d x(s)\right) m(d x) \\
& =\left[\frac{\lambda}{2 \pi}\right]_{\mathbb{R}^{n}}^{\frac{n}{2}} f\left(y\left(t_{1}\right) / \sqrt{2}+\left[\frac{\Delta_{1} a}{2}\right]^{\frac{1}{2}} v_{1}, \cdots, y\left(t_{n}\right) / \sqrt{2}+\sum_{j=1}^{n}\left[\frac{\Delta_{j} a}{2}\right]^{\frac{1}{2}} v_{j}\right) \\
& \cdot g\left(y\left(t_{1}\right) / \sqrt{2}-\left[\frac{\Delta_{1} a}{2}\right]^{\frac{1}{2}} v_{1}, \cdots, y\left(t_{n}\right) / \sqrt{2}-\sum_{j=1}^{n}\left[\frac{\Delta_{j} a}{2}\right]^{\frac{1}{2}} v_{j}\right) \\
& \cdot \exp \left\{-\frac{\lambda}{2}\left(v_{1}^{2}+\cdots+v_{n}^{2}\right)\right\} d \vec{v}
\end{aligned}
$$

Now let $u_{j}=\underset{k=1}{j}\left(\Delta_{k} a\right)^{\frac{1}{2}} v_{k}$ for $j=1, \cdots, n$. Then $v_{j}=\left(\Delta_{j} a\right)^{\frac{-1}{2}}\left(u_{j}-u_{j-1}\right)$ for $j=1, \cdots, n$ and so substituting into equation (5.4) we obtain equation (5.2) for $\lambda>0$. But the last expression in (5.4) is an analytic function of $\lambda$ throughout $\mathbf{C}_{+}$and so equation (5.2) is valid throughout $\mathbb{C}_{+}$.

The following theorem gives an interesting relationship involving generalized convolutions and generalized analytic Wiener integrals. 
THEOREM 5.1. Let $F$ and $G$ be as in Lemma 5.1. Then for all $\lambda \in \mathbb{C}_{+}$,

$$
\left(\mathrm{T}_{\lambda}(\mathrm{F} * \mathrm{G})_{\lambda}\right)(\mathrm{y})=\left(\mathrm{T}_{\lambda}(\mathrm{F})\right)(\mathrm{y} / \sqrt{2})\left(\mathrm{T}_{\lambda}(\mathrm{G})\right)(\mathrm{y} / \sqrt{2}) .
$$

PROOF. By Theorem 2.1, equation (5.5) holds for all $\lambda>0$. But the result now follows because $T_{\lambda}(F), T_{\lambda}(G)$ and $T_{\lambda}(F * G)_{\lambda}$ all have analytic extensions throughout $C_{+}$.

Our next lemma plays a key role in obtaining the existence of the GFFT of the GCP.

LEMMA 5.2. For all $\lambda \in \mathbb{C}_{+}^{\sim}$ let $\mathrm{H}(\lambda ; \overrightarrow{\mathrm{w}})$ be defined by (5.3) for $f \in \mathrm{L}_{\mathrm{p}_{1}}\left(\mathbb{R}^{\mathrm{n}}\right)$ and $\mathrm{g} \in \mathrm{L}_{\mathrm{p}_{2}}\left(\mathbb{R}^{\mathrm{n}}\right)$ with $1 \leq \mathrm{p}_{1} \leq 2$ and $1 \leq \mathrm{p}_{2} \leq 2$. Then for all $\lambda \in \mathbb{C}_{+}^{\sim}, \mathrm{H}(\lambda ; \cdot) \in \mathrm{L}_{\mathrm{r}}\left(\mathbb{R}^{\mathrm{n}}\right)$ where $\mathrm{r}$ is given by $\frac{1}{\mathrm{r}}=\frac{1}{\mathrm{p}_{1}}+\frac{1}{\mathrm{p}_{2}}-1$. In addition, if $\mathrm{r}=+\infty$ (ie, $\left.\mathrm{p}_{1}=\mathrm{p}_{2}=2\right)$, then $\mathrm{H}(\lambda ; \cdot) \in \mathrm{C}_{0}\left(\mathbb{R}^{\mathrm{n}}\right)$.

REMARK. Again when $\operatorname{Re} \lambda=0$, the integral in equation (5.3) is of course interpreted in the mean.

PROOF. If $1 \leq \mathrm{r}<+\infty$, then $\mathrm{H}\left(\lambda_{;} \cdot\right) \in \mathrm{L}_{\mathrm{r}}\left(\mathbb{R}^{\mathrm{n}}\right)$ by Proposition 26 in [11, p.317]. If $\mathrm{f}$ and $g$ are both in $L_{2}\left(\mathbb{R}^{n}\right)$ we first note that $H(\lambda ; \cdot)$ is in $L_{\infty}\left(\mathbb{R}^{n}\right)$ since for all $\vec{w} \in \mathbb{R}^{n}$,

$$
\begin{aligned}
|\mathrm{H}(\lambda ; \overrightarrow{\mathrm{w}})| & \leq|\lambda|^{\frac{\mathrm{n}}{2}} \gamma \int_{\mathbb{R}^{\mathrm{n}}}\left|\mathrm{f}\left[\frac{\overrightarrow{\mathrm{w}}+\overrightarrow{\mathrm{u}}}{\sqrt{2}}\right]\right|\left|\mathrm{G}\left[\frac{\overrightarrow{\mathrm{w}}-\overrightarrow{\mathrm{u}}}{\sqrt{2}}\right]\right| \mathrm{d} \overrightarrow{\mathrm{u}} \\
& \leq|\lambda|^{\frac{\mathrm{n}}{2}} \gamma\left\{\int_{\mathbb{R}^{\mathrm{n}}}\left|\mathrm{f}\left[\frac{\overrightarrow{\mathrm{w}}+\overrightarrow{\mathrm{u}}}{\sqrt{2}}\right]\right|^{2} \mathrm{~d} \overrightarrow{\mathrm{u}}\right\}^{\frac{1}{2}}\left\{\int_{\mathbb{R}^{\mathrm{n}}}\left|\mathrm{g}\left[\frac{\overrightarrow{\mathrm{w}}-\overrightarrow{\mathrm{u}}}{\sqrt{2}}\right]\right|^{2} \mathrm{~d} \overrightarrow{\mathrm{u}}\right\}^{\frac{1}{2}} \\
& =|\lambda|^{\frac{\mathrm{n}}{2}} \gamma(\sqrt{2})^{\mathrm{n}}\|\mathrm{f}\|_{2}\|\mathrm{~g}\|_{2} .
\end{aligned}
$$

A standard argument now shows that $\mathrm{H}\left(\lambda_{;} \cdot\right)$ belongs to $\mathrm{C}_{0}\left(\mathbb{R}^{\mathrm{n}}\right)$.

In our next theorem we show that the GFFT of the GCP is the product of transforms.

THEOREM 5.2. Let $F \in A_{n}^{\left(p_{1}\right)}$ and $G \in A_{n}^{\left(p_{2}\right)}$ with $1 \leq p_{1} \leq 2,1 \leq p_{2} \leq 2$ and $\frac{1}{\mathrm{p}_{1}}+\frac{1}{\mathrm{p}_{2}} \geq \frac{3}{2}$. Let $\mathrm{r}$ be given by $\frac{1}{\mathrm{r}}=\frac{1}{\mathrm{p}_{1}}+\frac{1}{\mathrm{p}_{2}}-1$. Then

$$
\left(T_{q}^{(r)}(F * G)_{q}\right)(y)=\left(T_{q}^{\left(p_{1}\right)}(F)\right)(y / \sqrt{2})\left(T_{q}^{\left(p_{2}\right)}(G)\right)(y / \sqrt{2})
$$

for all real $q \neq 0$.

PROOF. We first note that by Lemma $5.2,(F * G)_{q}$ is an element of $A_{n}^{(r)}$. Also, we note that $1 \leq \mathrm{r} \leq 2$ and so by Theorem 4.1, all three of the GFFT's in equation (5.6) exist. Equation (5.6) then follows from equation (5.5).

By choosing specific values of $\mathrm{p}_{1}$ and $\mathrm{p}_{2}$ in Theorem 5.2 we obtain the following corollary.

COROLLARY 5.1. i) Let $F, G \in A_{n}^{(1)}$. Then for all real $q \neq 0$, 


$$
\left(\mathrm{T}_{\mathrm{q}}^{(1)}(\mathrm{F} * \mathrm{G})_{\mathrm{q}}\right)(\mathrm{y})=\left(\mathrm{T}_{\mathrm{q}}^{(1)}(\mathrm{F})\right)(\mathrm{y} / \sqrt{2})\left(\mathrm{T}_{\mathrm{q}}^{(1)}(\mathrm{G})\right)(\mathrm{y} / \sqrt{2}) \text {. }
$$

ii) Let $F \in A_{n}^{(1)}$ and $G \in A_{n}^{(2)}$. Then for all real $q \neq 0$.

$$
\left(\mathrm{T}_{\mathrm{q}}^{(2)}(\mathrm{F} * \mathrm{G})_{\mathrm{q}}\right)(\mathrm{y})=\left(\mathrm{T}_{\mathrm{q}}^{(1)}(\mathrm{F})\right)(\mathrm{y} / \sqrt{2})\left(\mathrm{T}_{\mathrm{q}}^{(2)}(\mathrm{F})\right)(\mathrm{y} / \sqrt{2})
$$

iii) Let $F, G \in A_{n}^{\left(\frac{4}{3}\right)}$. Then for all real $q \neq 0$,

$$
\left(\mathrm{T}_{\mathrm{q}}^{(2)}(\mathrm{F} * \mathrm{G})_{\mathrm{q}}\right)(\mathrm{y})=\left(\mathrm{T}_{\mathrm{q}}^{\left(\frac{4}{3}\right)}(\mathrm{F})\right)(\mathrm{y} / \sqrt{2})\left(\mathrm{T}_{\mathrm{q}}^{\left(\frac{4}{3}\right)}(\mathrm{G})\right)(\mathrm{y} / \sqrt{2}) .
$$

We will finish this section by establishing a Parseval's relation for functionals $F$ and $G$ in $A_{n}^{(2)}$. In our proof we will use the ordinary Parseval's identity for $L_{2}\left(\mathbb{R}^{n}\right)$; that is to say

$$
\int_{\mathbb{R}^{n}} \hat{\mathbf{f}}(\overrightarrow{\mathrm{u}}) \overline{\overline{\mathrm{g}}}(\overrightarrow{\mathrm{u}}) \mathrm{du}=\int_{\mathbb{R}^{\mathrm{n}}} \mathrm{f}(\overrightarrow{\mathrm{u}}) \overline{\mathrm{g}}(\overrightarrow{\mathrm{u}}) \mathrm{d} \overrightarrow{\mathrm{u}}
$$

where $\hat{f}(\vec{u})=\int_{n} f(\vec{v}) e^{-2 \pi i\langle\vec{u}, \vec{v}\rangle} d \vec{v} \quad$ is the $n$-dimensional Fourier transform of $f$ and $\bar{g}$ is the complex conjugate of $\mathrm{g}$.

THEOREM 5.3. Let $F$ and $G$ be elements of $A_{n}^{(2)}$. Then the Parseval's identity

$$
\begin{gathered}
\int_{\mathrm{C}_{0}[0, \mathrm{~T}]}^{\operatorname{anf}}\left(\mathrm{T}_{\mathrm{q}}^{(2)}(\mathrm{F})\right)\left[\frac{\mathrm{Z}(\mathrm{x}, \cdot)}{\sqrt{2}}\right]\left(\mathrm{T}_{\mathrm{q}}^{(2)}(\mathrm{G})\right)\left[\frac{\mathrm{Z}(\mathrm{x}, \cdot)}{\sqrt{2}}\right] \mathrm{m}(\mathrm{dx}) \\
\quad=\int_{\mathrm{C}_{0}[0, \mathrm{~T}]}^{\operatorname{anf}} \mathrm{F}\left[\frac{\mathrm{Z}(\mathrm{x}, \cdot)}{\sqrt{2}}\right] \mathrm{G}\left[-\frac{\mathrm{Z}(\mathrm{x}, \cdot)}{\sqrt{2}}\right] \mathrm{m}(\mathrm{dx})
\end{gathered}
$$

holds for all real $q \neq 0$.

PROOF. a) For $\lambda>0$, we evaluate the Wiener integral below and obtain that

$$
\begin{aligned}
& \int_{C_{0}[0, T]} F\left[\frac{Z(x, \cdot)}{\sqrt{2 \lambda}}\right] G\left[-\frac{Z(x, \cdot)}{\sqrt{2 \lambda}}\right] m(d x) \\
& =\lambda^{\frac{n}{2}} \gamma \int_{\mathbb{R}^{n}} f(\vec{v} / \sqrt{2}) g(-\vec{v} / \sqrt{2}) \exp \left\{-\frac{\lambda}{2} \underset{j=1}{n} \frac{\left(v_{j}-v_{j-1}\right)^{2}}{\Delta_{j} a^{2}}\right\} d \vec{v} \\
& =(2 \lambda)^{\frac{n}{2}} \gamma \int_{\mathbb{R}^{n}} f(\vec{v}) g(-\vec{v}) \exp \left\{-\lambda \sum_{j=1}^{n} \frac{\left(v_{j}-v_{j-1}\right)^{2}}{\Delta_{j}^{a}}\right\} d \vec{v} .
\end{aligned}
$$

But since $f g \in L_{1}\left(\mathbb{R}^{n}\right)$, the above expression is an analytic function of $\lambda$ throughout $C_{+}$and a bounded continuous function of $\lambda$ on $\mathbb{C}_{+}^{-}$and so setting $\lambda=-$ iq we obtain that

$$
\int_{\mathrm{C}_{0}[0, \mathrm{~T}]}^{\operatorname{anf}_{\mathrm{q}}} \mathrm{F}\left[\frac{\mathrm{Z}(\mathrm{x}, \cdot)}{\sqrt{2}}\right] \mathrm{G}\left[-\frac{\mathrm{Z}(\mathrm{x}, \cdot)}{\sqrt{2}}\right] \mathrm{m}(\mathrm{dx})
$$


GENERALIZED TRANSFORMS AND CONVOLUTIONS

31

$$
=(-2 q i)^{\frac{n}{2}} \gamma \int_{\mathbb{R}^{n}} f(\vec{v}) g(-\vec{v}) \exp \left\{q i \underset{j=1}{\sum_{j=1}} \frac{\left(v_{j}-v_{j-1}\right)^{2}}{\Delta_{j}^{a}}\right\} d \vec{v}
$$

for all $q \in \mathbb{R}-\{0\}$.

b) We next note, using Lemma 4.2, that $\left(\mathrm{T}_{\mathrm{q}}^{(2)}(\mathrm{F})\right)(\mathrm{x} / \sqrt{2})\left(\mathrm{T}_{\mathrm{q}}^{(2)}(\mathrm{G})\right)(\mathrm{x} / \sqrt{2})$ is in $A_{n}^{(1)}$. Then, using Theorem 4.1, we see that for $\lambda>0$,

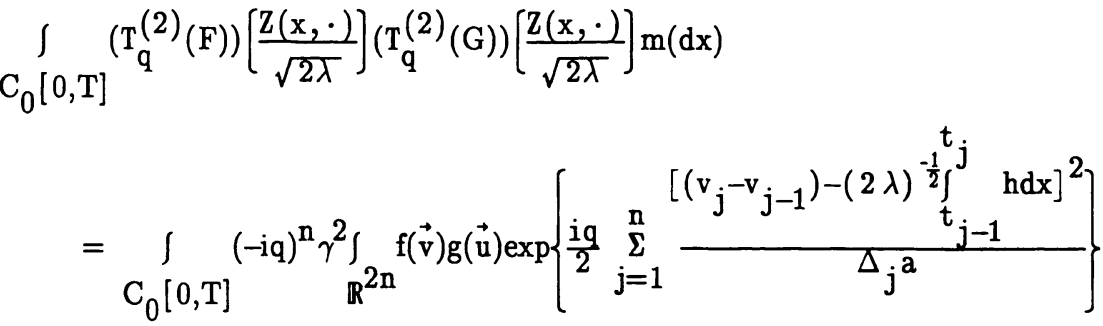

$$
\begin{aligned}
& \cdot \exp \left\{\frac{i q}{2} \sum_{j=1}^{n} \frac{\left[\left(u_{j}-u_{j-1}\right)-(2 \lambda)^{\frac{-1}{2}} \int_{j-1}^{t_{j}} h d x\right]^{2}}{\Delta_{j}{ }^{a}}\right\} d \vec{d} d \overrightarrow{v m}(d x) \\
& =(-i q)^{n} \lambda^{\frac{n}{2}} \gamma^{3} \int_{\mathbb{R}^{3 n}} f(\vec{v}) g(\vec{u}) \exp \left\{-\frac{\lambda}{2} \sum_{j=1}^{n} \frac{m_{j}^{2}}{\Delta_{j}^{a}}\right\} \\
& \text { - } \exp \left\{\frac{i q}{2} \sum_{j=1}^{n} \frac{\left[\left(v_{j}-v_{j-1}\right)-2^{-\frac{1}{2}} m_{j}\right]^{2}+\left[\left(u_{j}-u_{j-1}\right)-2^{-\frac{1}{2}} m_{j}\right]^{2}}{\Delta_{j}}\right\} d \vec{m} d \vec{u} d \vec{v} \text {. }
\end{aligned}
$$

Now letting $A(F, G, q)$ denote the left-hand side of equation (5.7) and setting $\lambda=-(-i q)$ in the above expression we obtain that

$$
\begin{aligned}
A(F, G, q) & =(-i q)^{n}(i q)^{\frac{n}{2}} \gamma^{3} \int_{\mathbb{R}^{3 n}} f(\vec{v}) g(\vec{u}) \exp \left\{-\frac{i q}{2} \sum_{j=1}^{n} \frac{m_{j}^{2}}{\Delta_{j}^{a}}\right\} \\
& \cdot \exp \left\{\frac{i q}{2} \sum_{j=1}^{n} \frac{\left.\left[\left(v_{j}-v_{j-1}\right)-2^{-\frac{1}{2}} m_{j}\right]^{2}+\left[\left(u_{j}-u_{j-1}\right)-2^{-\frac{1}{2}}\right]_{j}\right]^{2}}{\Delta_{j}{ }^{a}}\right\} d \vec{m} d \overrightarrow{u d v} .
\end{aligned}
$$

Next for $\mathrm{j}=1, \cdots, \mathrm{n}$ let $\mathrm{r}_{\mathrm{j}}=\mathrm{v}_{\mathrm{j}}-\mathrm{v}_{\mathrm{j}-1}$ and $l_{\mathrm{j}}=\mathrm{u}_{\mathrm{j}}-\mathrm{u}_{\mathrm{j}-1}$. Then

$$
\begin{array}{r}
A(F, G, q)=(-i q)^{n}(i q)^{\frac{n}{2}} \gamma^{3} \int_{\mathbb{R}^{3 n}} f\left[\sum_{j=1}^{1} r_{j}, \cdots, \sum_{j=1}^{n} r_{j}\right] g\left[\sum_{j=1}^{1} l_{j}, \cdots, \sum_{j=1}^{n} l_{j}\right] \\
\cdot \exp \left\{\frac{i q}{2} \sum_{j=1}^{n}\left[\frac{r_{j}^{2}+l_{j}^{2}-\sqrt{2} m_{j}\left(l_{j}+r_{j}\right)}{\Delta_{j} a^{2}}\right]\right\} d \vec{m} d \overrightarrow{l d} \vec{r} .
\end{array}
$$

Next let

$$
\begin{aligned}
& \mathrm{f}^{*}\left(\mathrm{r}_{1}, \cdots, \mathrm{r}_{\mathbf{n}}\right)=\mathrm{f}\left[\sum_{\mathrm{j}=1}^{1} \mathbf{r}_{\mathrm{j}}, \cdots, \sum_{\mathrm{j}=1}^{\mathrm{n}} \mathbf{r}_{\mathrm{j}}\right] \exp \left\{\frac{\mathrm{iq}}{2} \sum_{\mathrm{j}=1}^{\mathrm{n}} \frac{\mathrm{r}_{\mathrm{j}}^{2}}{\Delta_{\mathrm{j}} \mathrm{a}}\right\}, \\
& \mathrm{g}^{*}\left(l_{1}, \cdots, l_{\mathrm{n}}\right)=\overline{\mathrm{g}}\left[\sum_{\mathrm{j}=1}^{1} l_{\mathrm{j}}, \cdots, \sum_{\mathrm{j}=1}^{\mathrm{n}} l_{\mathrm{j}}\right] \exp \left\{-\frac{\mathrm{iq}}{2} \sum_{\mathrm{j}=1}^{\mathrm{n}} \frac{l_{\mathrm{j}}^{2}}{\Delta_{\mathrm{j}}^{\mathrm{a}}}\right\},
\end{aligned}
$$


and let $\mathrm{w}_{\mathrm{j}}=\frac{\mathrm{qm}_{\mathrm{j}}}{2 \pi \sqrt{2} \Delta_{j} \mathrm{a}}$ for $\mathrm{j}=1,2, \cdots, \mathrm{n}$. Then

$$
\begin{aligned}
& \mathrm{A}(\mathrm{F}, \mathrm{G}, \mathrm{q})=(-\mathrm{iq})^{\mathrm{n}}(\mathrm{iq})^{\frac{\mathrm{n}}{2}} \gamma^{3}\left(2 \pi \sqrt{2} \Delta_{\mathrm{j}} \mathrm{a}\right)^{\mathrm{n}} \mathrm{q}^{-\mathrm{n}} \int_{\mathbb{R}^{\mathrm{n}}} \int_{\mathbb{R}^{\mathrm{n}}} \mathrm{f}^{*}(\overrightarrow{\mathrm{r}}) \exp \left\{-2 \pi \mathrm{i} \sum_{\mathrm{j}=1}^{\mathrm{n}} \mathrm{r}_{\mathrm{j}} \mathrm{w}_{\mathrm{j}}\right\} \mathrm{dr} \\
& \cdot \int_{\mathbb{R}^{\mathrm{n}}} \overline{\mathrm{g}^{*}}(\vec{l}) \exp \left\{-2 \pi \mathrm{i} \sum_{\mathrm{j}=1}^{\mathrm{n}} l_{\mathrm{j}} \mathrm{w}_{\mathrm{j}}\right\} \mathrm{d} \vec{l} \mathrm{~d} \overrightarrow{\mathrm{w}} \\
& =(-2 q i)^{\frac{n}{2}} \gamma \int_{\mathbb{R}^{n}} \widehat{f^{*}}(\vec{w}) \mathrm{g}^{*}(-\vec{w}) d \vec{w} \\
& =(-2 q i)^{\frac{n}{2}} \gamma \int \mathrm{f}^{*}(\vec{w}) \overline{g^{*}}(-\vec{w}) d \vec{w} \\
& \mathbb{R}^{n} \\
& =(-2 q i)^{\frac{n}{2}} \gamma \int_{\mathbb{R}^{n}} f\left(\sum_{j=1}^{1} w_{j}, \cdots, \sum_{j=1}^{n} w_{j}\right] g\left[-\sum_{j=1}^{1} w_{j}, \cdots,-\sum_{j=1}^{n} w_{j}\right] \exp \left\{i q \underset{j=1}{n} \frac{w_{j}^{2}}{\Delta j_{j}^{a}}\right\} d \vec{w} \\
& =(-2 q i)^{\frac{n}{2}} \gamma \int_{\mathbb{R}^{n}} f(\vec{v}) g(-\vec{v}) \exp \left\{q i \sum_{j=1}^{n} \frac{\left(v_{j}-v j-1\right)^{2}}{\Delta_{j}^{a}}\right\} d \vec{v}
\end{aligned}
$$

for all real $q \neq 0$, which in view of (5.8) establishes (5.7).

ACKNOWLEDGEMENT. The third author expresses his graditude to the University of Nebraska for a Faculty Development Fellowship as well as the hospitality of Idaho State University where the research was carried out.

\section{REFERENCES}

1. BRUE, M.D. A Functional Transform for Feynman Integrals Similar to the Fourier Transform, Doctoral Thesis, University of Minnesota, (1972).

2. CAMERON, R.H. and STORVICK, D.A. An $\mathrm{L}_{2}$ analytic Fourier-Feynman Transform, Michigan Math. J. 23(1976), 1-30.

3. JOHNSON, G.W. and SKOUG, D.L. An $\mathrm{L}_{\mathrm{p}}$ Analytic Fourier-Feynman Transform, Michigan Math. J. 26(1979), 103-127.

4. HUFFMAN, T., PARK, C., and SKOUG, D. Analytic Fourier-Feynman Transforms and Convolution, Trans. Amer. Math. Soc. 347(1995), 661-673.

5. HUFFMAN, T., PARK, C. and SKOUG, D. Convolution and Fourier-Feynman Transforms, submitted for publication.

6. CHUNG, D.M., PARK, C. and SKOUG, D. Generalized Feynman Integrals via Conditional Feynman Integrals, Michigan Math. J. 40(1993), 377-391.

7. PARK, C. and SKOUG, D. Generalized Feynman Integrals: the $\mathscr{L}\left(\mathrm{L}_{2}, \mathrm{~L}_{2}\right)$ Theory, to appear in Rocky Mountain J. of Math.

8. CAMERON, R.H. and STORVICK, D.A. Some Banach algebras of Analytic Feynman Integrable Functionals, in Analytic Functions, Kozubnik, 1979, Lecture Notes in Math. Vol. 798, Springer-Verlag, Berlin, 1980, 18-67.

9. CHANG, K.S. Scale-invariant Measurability in Yeh-Wiener Space, J. Korean Math. Soc. 19 (1982), 61-67.

10. JOHNSON, G.W. and SKOUG, D.L. Scale-Invariant Measurability in Wiener Space, Pacific J. Math. 83(1979), 157-176.

11. ROYDEN, H.L. Real Analysis, Macmillan, 1988. 


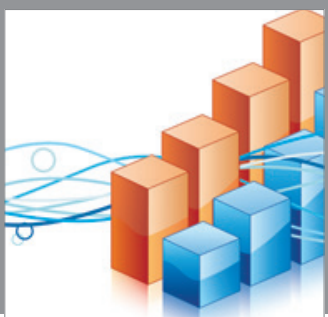

Advances in

Operations Research

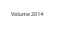

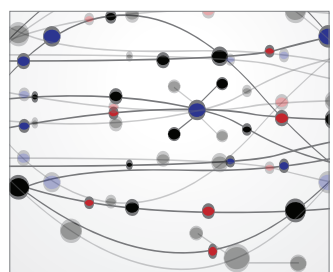

\section{The Scientific} World Journal
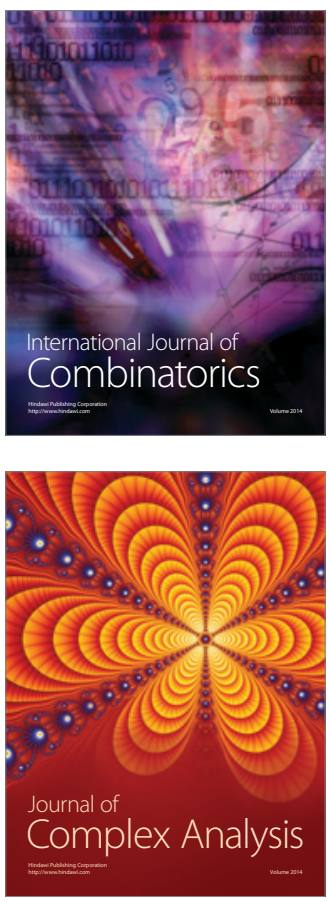

International Journal of

Mathematics and

Mathematical

Sciences
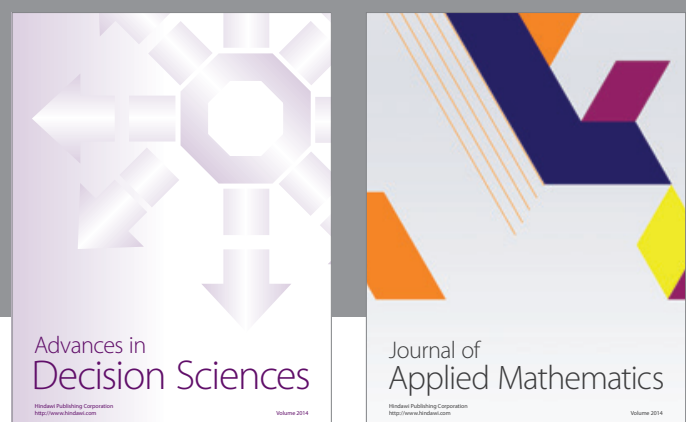

Journal of

Applied Mathematics
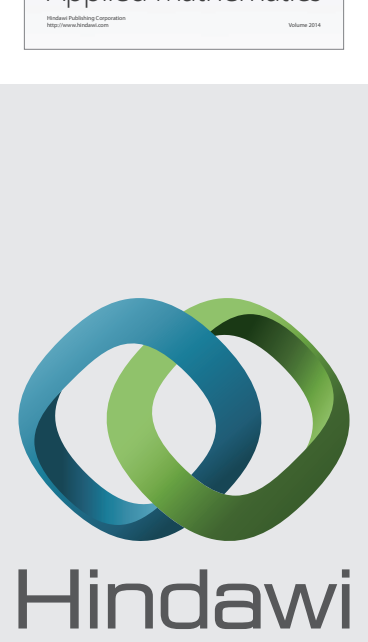

Submit your manuscripts at http://www.hindawi.com
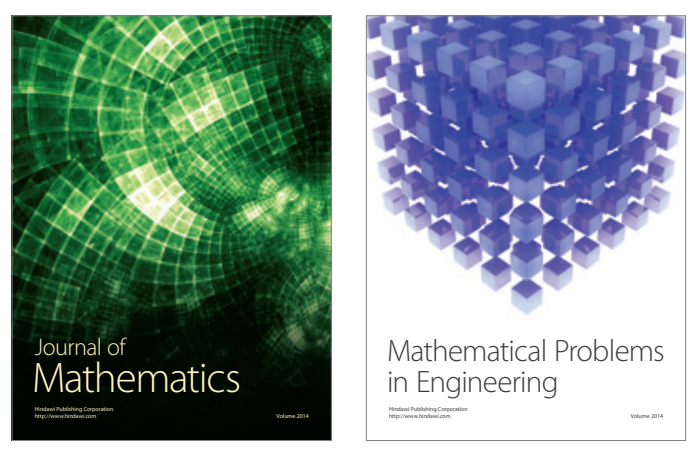

Mathematical Problems in Engineering
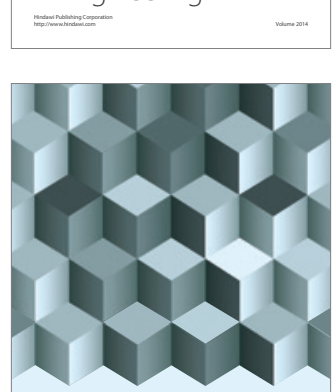

Journal of

Function Spaces
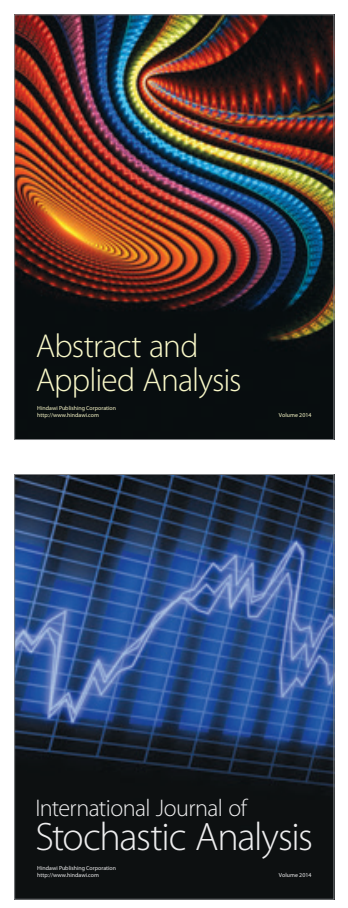

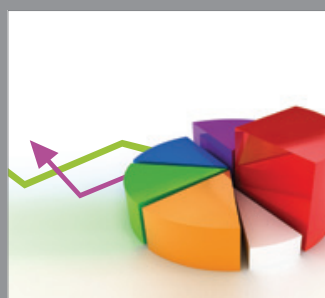

ournal of

Probability and Statistics

Promensencen
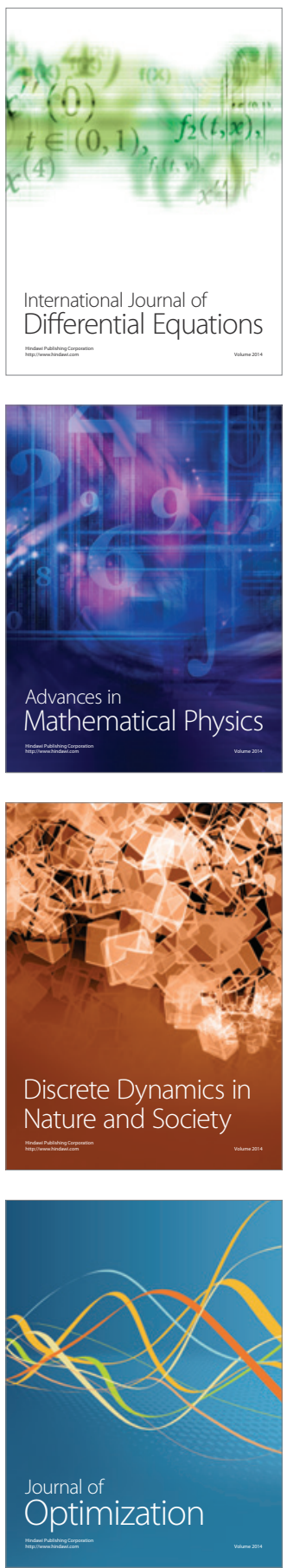